\title{
Resultados a longo prazo com o uso do implante coclear em crianças: Revisão sistemática
}

\section{Long-term results by using cochlear Implants on children: Systematic review}

\author{
Liège Franzini Tanamati', Orozimbo Alves Costa², Maria Cecilia Bevilacqua ${ }^{3}$. \\ 1) Doutoranda em Fisiopatologia dos Distúrbios da Comunicação da Faculdade de Medicina da Universidade de São Paulo - FMUSP. Fonoaudióloga do Núcleo do Ouvido \\ Biônico do Hospital Samaritano de São Paulo. \\ 2) Professor Titular da Universidade de São Paulo. Coordenador do Núcleo do Ouvido Biônico do Hospital Samaritano de São Paulo \\ 3) Professora Titular da Universidade de São Paulo. Coordenadora do Centro de Pesquisas Audiológicas do Hospital de Reabilitação de Anomalias Craniofaciais e \\ Coordenadora do Núcleo do Ouvido Biônico do Hospital Samaritano de São Paulo. \\ Instituição: Faculdade de Medicina da Universidade de São Paulo - FMUSP. \\ São Paulo/SP - Brasil. \\ Endereço para correspondência: Liège Franzini Tanamati - Rua Cincinato Braga, 59. Conjunto 5d1 -Bairro: Paraíso - São Paulo / SP - Brasil - CEP: 01333-011 - Telefone: \\ (+55 11) 3288-4186/3262-0675 - E-mail 1: liegeft@hotmail.com E-mail 2: liege_tanamati@yahoo.com.br E-mail 3: liegeft@usp.br \\ Artigo recebido em 19 de Janeiro de 2011. Artigo aprovado em 9 de Março de 2011.
}

\section{RESUMO}

Introdução: Os benefícios do Implante coclear (IC) nos primeiros anos de uso em crianças já foram detalhadamente descritos na literatura. Porém, são escassos os estudos que relatam os resultados após longos períodos de uso do dispositivo.

Objetivo: Revisar a evidência disponível na literatura para responder ao questionamento: "O Implante Coclear contribuiu de modo efetivo para o desenvolvimento das habilidades comunicativas em adolescentes e adultos jovens que cresceram usando este dispositivo?".

Método: Para responder ao questionamento proposto, foi realizada a revisão sistemática da literatura. Diversas bases de dados eletrônicas e anais de congressos foram selecionados para localizar a evidência referente aos resultados do uso prolongado do IC. Os títulos potencialmente relevantes para responder ao objetivo da revisão sistemática foram recuperados e revisados usando um protocolo de análise dos dados. Para cada artigo incluído na presente revisão sistemática foi realizada a resenha em uma ficha protocolar e a classificação segundo o nível de evidência e grau de recomendação.

Síntese dos Dados: Quinze estudos contemplaram os critérios e foram incluídos na revisão sistemática. Os resultados descritos mostraram que a primeira geração de crianças implantadas alcançou níveis de competência linguística e acadêmica similares aos indivíduos com audição normal da mesma idade.

Comentários Finais: O IC foi contribuiu de maneira efetiva para o desenvolvimento de habilidades comunicativas funcionais em adolescentes e jovens adultos que cresceram usando o dispositivo eletrônico.

Palavras-chave: implante coclear, avaliação de resultados (cuidados de saúde), seguimentos.

\section{SUMMARY}

Introduction: Cochlear Implant (CI) benefits given to children in the first years of usage have been thoroughly described in literature. Nonetheless, studies reporting the results after the device has been long used are scarce.

Objective: Review the available evidence in literature to answer the query: "Did Cochlear Implant effectively contribute to develop the communication abilities in children and young adults who grew up with this device?

Method: To answer the suggested query, a systematic literature review was performed. Several electronic databases and congress records have been selected to find the evidence regarding the long-term CI results. The likely significant titles to answer the objective of the systematic review were retrieved and reviewed by using a data analysis protocol. For every article encompassed in the present systematic review, short notes were made on a filing card as well as the classification, according to evidence level and degree of recommendation. Data synthesis: Fifteen studies fulfilled the requirements and were included in the systematic review. The achieved results showed that the first generation of implanted children reached levels of linguistic and academic proficiency similar to normal hearing individuals at the same age.

Final Comments: CI effectively contributed to develop the functional communication abilities in children and young adults who grew up with this electronic device.

Keywords: cochlear implant, evaluation of results (health care), follow-up studies. 


\section{INTRODUÇÃO}

Em 1990, o FDA (Food and Drug Admuistration) aprovou a realização do Implante Coclear (IC) em crianças a partir dos dois anos de idade (1). Desde então, paralelamente ao cenário de expansão dos critérios de indicação do IC na população pediátrica e ao desenvolvimento tecnológico aplicado na fabricação dos dispositivos, um número crescente de crianças tem sido implantada, e com isso, pesquisas e estudos clínicos contínuos são conduzidos no sentido de mensurar os resultados proporcionados pelo IC, especialmente, em função das características de cada usuário e do tempo de uso do dispositivo.

O IC é considerado o recurso tecnológico mais efetivo para o tratamento da deficiência auditiva prélingual neurossensorial de grau severo a profundo disponível atualmente. Trata-se de uma intervenção cujos efeitos e resultados para as habilidades comunicativas em crianças são obtidos ao longo dos anos (2).

Resultados a curto prazo, mensurados durante os primeiros anos de uso do IC em crianças com deficiência auditiva pré-lingual, têm sido detalhadamente descritos na literatura. De maneira geral, os resultados destes estudos demonstraram benefícios inquestionáveis com o uso do IC, sejam eles no âmbito da linguagem receptiva ou expressiva, no processo de aprendizado acadêmico ou nas áreas afetiva, social e emocional. No entanto, apesar de haver um consenso, principalmente no que diz respeito à melhora na percepção dos sons ambientais, na fala, bem como em outras áreas do desenvolvimento linguístico, há concordância entre os estudos conduzidos nos primeiros anos de uso do IC quanto à existência de variabilidade de resultados observados nas crianças implantadas. Diversos fatores descritos na literatura, tais como, a etiologia, a idade na implantação, a presença de audição residual, a reabilitação auditiva, a participação familiar no processo terapêutico, podem potencialmente contribuir para a variabilidade no desempenho das crianças usuárias de IC $(3,4,5)$.

Porém, não se sabe ao certo se esta variabilidade nos resultados obtidos em crianças com pouco tempo de uso do dispositivo permanecerá após o uso do IC a médio e a longo prazo, nem tampouco, se benefícios satisfatórios serão alcançados pelos usuários de IC após muitos anos de uso do dispositivo, tendo como referência seus pares com audição normal. Escassos são os estudos que realizaram a avaliação dos resultados após 7 anos de uso (6).

No decorrer do uso do dispositivo de IC e do processo terapêutico, as famílias e os profissionais que trabalham com as crianças implantadas podem se deparar com situações e resultados inusitados, permeados por dúvidas e até insatis- fações. Nem todas as crianças implantadas são capazes de alcançar os resultados esperados pela família nos primeiros anos de uso do dispositivo. Diversos fatores podem interferir neste processo de aquisição das habilidades auditivas e de linguagem por meio do IC, e por fim, acarretar um atraso na aquisição destas habilidades e, em certos casos, na impossibilidade da criança alcançar níveis de habilidades comunicativas apropriadas para a idade (7).

Além disso, a evolução das habilidades de audição e de linguagem oral e o ritmo do desenvolvimento destas habilidades não são os mesmos para todas as crianças $(8,9,10,11)$. Até que nível de habilidade comunicativa a criança irá alcançar e, em quanto tempo irá ocorrer, ou ainda, se as crianças implantadas em idades maiores conseguirão, ao longo do tempo, alcançar os mesmos resultados das crianças implantadas em idades menores, são questionamentos cujas respostas não são obtidas logo nos primeiros anos de uso do dispositivo pela criança (12).

Outro fator importante a ser considerado em relação à importância dos estudos a longo prazo, diz respeito às informações relacionadas à funcionalidade do dispositivo e as possíveis intercorrências após anos de uso. Durante o processo de decisão pela cirurgia, bem como de escolha do modelo e da marca do IC, informações sobre a integridade dos dispositivos provindos de dados históricos ou por meio de avaliações mais atuais são fundamentais e devem ser fornecidas em um formato transparente, padronizado e de fácil compreensão, de maneira a auxiliar os profissionais envolvidos no aconselhamento, os candidatos ao IC e os membros da família (13).

Os estudos com crianças após longos períodos de uso do IC representam uma maneira de nortear os profissionais e as famílias tanto no que se refere ao processo terapêutico e ao aconselhamento sobre as expectativas como também no sentido de melhor compreender os fatores envolvidos nos processos de desenvolvimento das habilidades comunicativas, acadêmicas e ocupacionais de crianças que crescerão usando o IC. À medida que verificam as principais dificuldades não supridas ao longo do tempo, podem nortear os profissionais a realizarem uma intervenção terapêutica anterior, de modo a contribuir para maior adaptação a sociedade $(6,14,15,16)$.

Considerando a importância dos estudos a longo prazo com as crianças usuárias de IC, o objetivo deste artigo foi verificar se, após vários anos de uso do IC, foram obtidos desfechos satisfatórios no que se refere às habilidades comunicativas de adolescentes e jovens adultos que cresceram usando o dispositivo eletrônico. A revisão sistemática da literatura foi conduzida no intuito de reunir a evidência científica disponível na literatura para responder ao questionamento clínico. Este trabalho foi aprovado pela 
Comissão de Ética para Análise de Projetos de Pesquisa CAPPesq com o protocolo de pesquisa no. 0685/08.

\section{Metodologia da ReVISÃo SISTEMÁTICA DA LITERATURA}

Um dos itens fundamentais a metodologia da revisão sistemática é a formulação da pergunta da pesquisa. Considerando o objetivo da revisão sistemática do presente estudo, a pergunta formulada foi: O Implante Coclear contribuiu de modo efetivo para o desenvolvimento das habilidades comunicativas em adolescentes e adultos jovens que cresceram usando este dispositivo?"

\section{Critérios de seleção dos estudos}

Nesta etapa, após a formulação da pergunta, a busca de evidência teve início com a determinação dos critérios de seleção dos estudos primários. A partir destes critérios, espera-se que todos os artigos importantes ou que possam ter algum impacto na conclusão da revisão sistemática sejam encontrados e incluídos (17).

\section{Tipos de Estudos}

Foram selecionados para a análise, os estudos publicados nos últimos dez anos, ou seja, entre 2000 e 2010, nos idiomas: inglês, espanhol ou português. Quanto ao nível de evidência científica, devido a escassez de estudos com nível de evidência 1 e 2 na área de Audiologia, também foram selecionados para a revisão sistemática do presente estudo, os artigos com nível de evidência 3, 4 e 5 (18). A Tabela 1 mostra os níveis de evidência científica, adaptados por Cox (19) para a área de Audiologia.

\section{Participantes}

Uma vez que o objetivo da presente revisão sistemática foi avaliar os resultados a longo prazo do IC realizado em crianças, o principal critério de inclusão referente ao tempode uso do dispositivo contemplou os estudos conduzidos com adolescentes ou jovens adultos com, no mínimo, sete anos de uso. No que se refere à idade na cirurgia e a época de instalação da deficiência auditiva, os estudos com grupos de deficientes auditivos pré-linguais que realizaram a cirurgia na adolescência ou na fase adulta não foram aceitos dentro dos critérios de inclusão. Estudos com grupo de adultos com deficiência auditiva pós-lingual também foram excluídos.

\section{Intervenção}

A metodologia e os testes empregados nos estudos com o objetivo de avaliar o desempenho dos usuários de
Tabela I. Avaliação do nível de evidência.

\begin{tabular}{|c|c|}
\hline Nive & \\
\hline I & $\begin{array}{l}\text { Revisões sistemáticas e meta análises de estudos } \\
\text { clínicos aleatórios ou outros estudos de qualidade. }\end{array}$ \\
\hline 2 & Estudos clínicos aleatórios controlados. \\
\hline 3 & Estudos de intervenção não aleatórios. \\
\hline 4 & $\begin{array}{l}\text { Estudo de coorte; estudo de caso-controle, estudos } \\
\text { seccionais cruzados e experimen-tos não } \\
\text { controlados. }\end{array}$ \\
\hline 5 & Estudos de caso. \\
\hline 6 & Opiniões de especialistas. \\
\hline
\end{tabular}

Tabela 2. Descritores no idiomainglês encontrados no DeCS e a correspondência para o português.

\begin{tabular}{l|l}
\hline DeCS - Inglês & DeCS - Português \\
\hline Cochlear/mplantation, Child, & Implante Coclear, Criança, \\
AdolescentYoungadult, & Adolescente,Adulto jovem, \\
SpeechPerception, Speech & Percepção dafala, \\
Intelligibility, Language, & Inteligibilidadedafala \\
$\begin{array}{l}\text { OutcomeAssessmentFollow- } \\
\text { upstudies, Educationalstatus. }\end{array}$ & $\begin{array}{l}\text { Linguagem,Avaliaçãode } \\
\text { resultados, Seguimentos } \\
\end{array}$ \\
\hline
\end{tabular}

IC não foram considerados critérios de exclusão. Foram selecionados os estudos cuja intervenção tenha sido realizada em um grupo de usuários com mais de 7 anos de uso do IC, respeitando os demais critérios de seleção relacionados aos participantes.

\section{Desfechos clínicos}

Para a avaliação da efetividade do dispositivo de Implante coclear após anos de uso, os desfechos clínicos considerados relevantes à pergunta da presente revisão sistemática incluíram as seguintes categorias de interesse: resultados expressos em porcentagem de acertos nos testes de percepção e de inteligibilidade da fala, resultados expressos por meio da classificação de escalas do desenvolvimento das habilidades auditivas, de linguagem e de inteligibilidade da fala e descrição da situação acadêmica e/ ou profissional e do uso do dispositivo.

\section{Localização dos estudos}

\section{Descritores}

Os descritores ou as palavras-chave utilizados para localização dos estudos relevantes para responder a pergunta formulada na presente revisão sistemática constam na Tabela 2. 


\section{Estratégia de busca}

A estratégia de busca é a sintaxe da estratégia usada para o levantamento bibliográfico nas bases de dados. Para o presente estudo, foi elaborada uma estratégia de busca especifica, empregando os descritores em grupos, com no mínimo, duas palavras-chave: cochlearimplantation AND child OR adolescent OR young adult; cochlearimplantation AND follow-up studies OR Outcomes. Termos adicionais também foram utilizados: cocblear implantation AND speech perception OR speech intelligibility OR language OR educational status.

\section{Fontes de estudo}

Para o levantamento bibliográfico dos possíveis artigos a serem incluídos na revisão sistemática, as seguintes fontes de pesquisa foram consultadas:

- Bases eletrônicas de dados: A busca por estudos primários foi então realizada nas bases eletrônicas de dados: Lilacs, Medline, Scielo, Cochrane Library, Pubmed, Embase, Institute for Scientific Information (ISI) e Science Direct. A última busca manual realizada nas bases eletrônicas de dados ocorreu em setembro/2010. Após esta data, o serviço de "alerta eletrônico", disponível nas bases de dados, foi acionado.

- Anais de Congressos Nacionais: no país, destaca-se por seu histórico e por sua importância no cenário científico nacional, o Encontro Internacional de Audiologia (EIA). Até o ano de 2008, a busca foi realizada de maneira manual, nos anais impressos do evento. Após 2008, os anais dos eventos, disponíveis no site http://www.audiologiabrasil.org.br, foram acessados.

- Outras bases de dados: considerando a importância de se realizar o levantamento bibliográfico para a revisão sistemática em diferentes tipos de bases de dados, a busca em sites específicos de dissertações e teses (nacionais e internacionais) foi realizada nos bancos de informações digitais: Digital Dissertation Abstracts, Biblioteca Digital Brasileira de Teses e Dissertações, Biblioteca Digital de Teses e Dissertações da USP.

Identificação, seleção, extração dos dados e inclusão dos estudos

A partir da aplicação da estratégia de busca contendo os descritores definidos, foi realizada a identificação dos títulos nas diferentes bases eletrônicas de dados.

No intuito de não correr o risco de excluir estudos importantes para a revisão, após reunião de consenso, dois revisores selecionaram todos os títulos identificados, acompanhados ou não do resumo, potencialmente relevantes ao objeto de estudo. Após a seleção dos títulos relevantes
Tabela 3. Grau de recomendação.

A Estudos de níveis I ou 2 com conclusões consistentes.

B Estudos de níveis 3 ou 4 com conclusões consistentes ou evidênciaextrapolada(generalizado paraumasituação naqual não é totalmente relevante) de estudos de níveis I ou 2.

C Estudos de nível 5 ou evidência extrapolada (generalizado para uma situação na qual não é totalmente relevante) de estudos 3 e 4.

D Estudos de nível6. Estudos inconsistentes ou inconclusivos de quaisquerníveis. Qualquerestudo que apresente umalto risco de viés.

realizou-se a recuperação dos artigos na íntegra e avaliação de cada artigo mediante protocolo contendo os tópicos: tipo de estudo, participantes, intervenção adotada, desfechos encontrados.

Os estudos incluídos contemplaram os critérios de elegibilidade definidos no início do protocolo metodológico da presente revisão, no sentido de responderem à pergunta que norteia esta revisão sistemática. Os principais dados de cada artigo recuperado foram detalhadamente coletados por meio de uma ficha protocolar padronizada para o presente estudo.

Análise e apresentação dos dados

Na presente revisão sistemática, após o preenchimento da ficha protocolar contendo as informações detalhadas de cada artigo, os artigos foram então classificados quanto: à qualidade da evidência (Tabela 3) e ao nível de evidência científica (Tabela 1), adaptados por Cox (19) para a área de Audiologia.

No que se refere à apresentação dos dados, as informações relevantes de cada artigo, bem como, a classificação quanto ao grau de recomendação foram reunidos em tabelas para facilitar a consulta e o acesso durante a apresentação e discussão dos resultados.

\section{RESULTADOS DA REVISÃO DA LITERATURA}

\section{Quanto a seleção dos estudos potencialmen- te relevantes para a revisão sistemática}

\section{Resultados nas bases eletrônicas de dados}

A partir da estratégia de busca nas diferentes bases eletrônicas de dados, foram identificados 282 títulos, com ou sem resumo, potencialmente relevantes para o objeto de estudo da presente revisão sistemática. Os títulos identifica- 
dos em cada uma das bases de dados estão descritos na Tabela 4 .

Dentre os 282 títulos, 82 apareceram em mais de um banco eletrônico de dados. Os 197 títulos duplicados foram descartados, restando um total de 85 títulos potencialmente relevantes a revisão sistemática.

\section{Resultados em Anais de Congressos}

A partir do levantamento manual e digital referente aos anais do Encontro Internacional de Audiologia realizados entre 1985 a 2010 não foram encontrados estudos potencialmente relevantes a presente revisão sistemática.

\section{Resultados em outras bases de dados}

Além da busca na base eletrônica de dados por artigos científicos, a pesquisa bibliográfica nas bases digitais de dissertações e teses também foi conduzida. A partir desta busca, foram selecionadas duas teses, encontradas no "Digital Dissertation Abstracts".

\section{Quanto à análise dos estudos selecionados}

\section{Bases eletrônicas de dados}

Dentre os 85 títulos selecionados para recuperação dos artigos, somente um não pode ser recuperado, por não estar disponível para o acesso eletrônico no país. (Svirsky et al., 2007. "The effects of age at implantation on speech intelligibility in pediatric cochlearimplant users: Clinical outcomes and sensitive periods. "). Por esta razão, no total, foram recuperados 84 artigos.

Após leitura dos artigos completos, apenas 15 contemplaram os critérios de elegibilidade deste estudo e foram incluídos na revisão sistemática da literatura. Sessenta e nove artigos não foram incluídos por apresentarem um ou mais fatores de exclusão. A Tabela 5 apresenta os critérios que levaram a exclusão dos artigos recuperados e em quantos artigos o critério de exclusão ocorreu.

As referências completas dos 15 artigos incluídos na revisão sistemática e os principais resultados coletados a partir da ficha protocolar constam descritos nas Tabelas 6 e 7 , respectivamente.

\section{Outras bases de dados}

Duas teses encontradas no "Digital Dissertation Abstracts"foram selecionadas para análise por a presentarem títulos potencialmente relevantes ao objeto de estudo da presente revisão sistemática: "The experience of
Tabela 4. Distribuiçãa dos estudos identificados por base de dados.

\begin{tabular}{ll}
\hline Trabalhos Identificados & $N=282$ \\
\hline Lilacs & $N=1$ \\
Medline & $N=78$ \\
Cochrane & $N=0$ \\
Scielo & $N=1$ \\
ISI & $N=69$ \\
Pubmed & $N=79$ \\
S. Direct & $N=17$ \\
Embase & $N=37$ \\
\hline
\end{tabular}

Tabela 5. Razões para exclusão dos artigos.

\begin{tabular}{lc}
\hline Razão da exclusão & $\begin{array}{c}\text { Número de artigos } \\
\text { excluídos }\end{array}$ \\
\hline Tempo de uso do Implante coclear & 4 I \\
Não constava o tempo de uso do & 9 \\
Implante coclear & $\mid$ | \\
Idade na avaliação & 3 \\
Adultos pós-linguais & 3 \\
Adolescentese jovensadultos pré-linguais & 19 \\
Objetivo do estudo & 13 \\
Metodologia e/ou resultados escassos ou & 2 \\
pouco detalhados & \\
Opinião de especialista/Revisão de literatura & \\
\hline
\end{tabular}

adolescents with cochlear implants" (Kops KE, 2003) e "Perception and production of speech intonation in pediatric cochlear implant recipients and children with normal bearing" (PENG SC, 2005).

Porém, ambas foram excluídas após avaliação detalhada do trabalho por não contemplarem os critérios de seleção da presente revisão sistemática quanto ao objeto de estudo, ao tempo de uso do IC, a idade na cirurgia e a época de instalação da deficiência auditiva.

\section{DISCUSSÃO}

Considerando a importância em conhecer os resultados obtidos a médio e a longo prazo e a escassez deste tipo de estudo com a população pediatria usuária de IC, o objetivo deste trabalho foi verificar, por meio da revisão sistemática da literatura, a efetividade do IC para o desenvolvimento das habilidades comunicativas de adolescentes e jovens adultos que cresceram usando o dispositivo eletrônico. 
Tabela 6. Referências Bibliográficas dos estudos incluídos na revisão sistemática.

Artigos Incluídos

I TylerRS.7-year speech perception results and the effects of age, residual hearing and preimplant speech perception in prelingually deaf children using the Nucleus and Clarion cochlear implants. Adv Otorhinolaryngol. 2000, 57:305- 10.

2 WaltzmanSB, Cohen NL, GreenJ, RolandJT. Long-termeffects of cochlearimplants inchildren. Otolaryngol Head NeckSurg. 2002, |26(5):505-51।.

3 Manrique M, Cervera-PazFJ, HuarteA, MolinaM. Prospective long-termauditory results of cochlear implantation in prelinguistically deafened children: the importance of early implantation. Acta Otolaryngol Suppl. 2004, 552:55-63.

$4 \quad$ PengSC, SpencerLJ, TomblinJB. Speech intelligibility of pediatric cochlear implantrecipients with 7 years of device experience. J Speech Lang Hear Res. 2004, 47(6): I227- 1236.

5 SpencerL); GantzB]; KnutsonJF. Outcomesandachievementofstudentswhogrewupwithaccessto cochlearimplants. Laryngoscope. 2004, I |4(9):|576-158|.

6 BeadleEA etal. Longtermfunctional outcomesandacademic-occupational status in implanted children after $\mid 0$ to $\mid 4$ years of cochlear implant use. Otol Neurotol. 2005, 26(6): I I 52- I 160.

7 HaenselJ, EngelkeJC, OttenjannW, WesthofenM.. Long-termresults of cochlear implantinchildren. Otolaryngol Head NeckSurg. 2005, 132(3):456-8.

8 ConnorCM. Examiningthe communication skills of ayoung cochlear implant pioneer.J DeafStud DeafEdu. 2006, I I (4):449-460.

9 UzielAS, etal. Ten-yearfollow-up of a consecutive series of children with multichannel cochlear implants. Otol Neurotol. 2007, 28(5):615-628.

10 GeersAE,TobeyE,MoogJ, BrennerC. Long-termoutcomes of cochlearimplantation in the preschoolyears:fromelementarygrades to high school. Int J Audiol. 2008, 47 (Suppl 2):S2 I-S30.

I I Colletti L. Long-termfollow-up of infants (4- I I months) fitted with cochlear implants. Acta Otolaryngol. 2009 Apr, | 29(4):36 | -6.

12 Said, TC, Bevilacqua MC, MoretALM. Speech perception in pre-lingual deaf users of cochlear implant. Pró-Fono. 20 I0, 22(3):2759.

I3 Davidson LS, GeersAE, BrennerC. Cochlearimplantcharacteristicsand speech perceptionskills of adolescents with long-term device use. Otol Neurotol. 20 10, 31: 1310-14.

I HabibMG, WaltzmanSB, TajudeenB, SvirskyMA. Speech production intelligibility of early implanted pediatric cochlear implant users. Int Pediatr Otorhinolaryngol. 20 I0, 74(8):855-859.

I5 Venail F, VieuA, ArtieresF, Mondain M, UzielA. Educationalandemploymentachievements in prelingually deafchildren who receive cochlearimplants. Arch Otolaryngol Head NeckSurg. 20 I0, I36(6):575.

Um número predominante de estudos na literatura avaliou os usuários durante, no máximo, seis anos de uso do dispositivo. Ao final do levantamento bibliográfico, apenas um grupo de 15 artigos, sendo todos estudos primários, foram selecionados para apreciação, por contemplaram os critérios de inclusão para responder a pergunta da presente revisão sistemática. Dentre os artigos incluídos, 8 eram estudos prospectivos, 2 retrospectivos, 4 transversais e 1 estudo de caso longitudinal. Os estudos apresentaram grau de recomendação C e D.

O primeiro estudo avaliando os benefícios do IC a longo prazo em crianças foi realizado por TyLER (2000). Na época, somente estudos com crianças implantadas durante cinco anos após a cirurgia havia sido conduzidos. O autor verificou as possíveis variáveis que poderiam contribuir para os diferentes níveis de benéficos proporcionados pelo
IC para o desenvolvimento das habilidades auditivas durante os sete primeiros anos de uso do dispositivo. A partir de então, outros estudos a longo prazo foram conduzidos na literatura internacional. No entanto, considerando que o FDA aprovou a indicação do IC em crianças a partir de dois anos de idade, no ano de 2010, completando 20 anos do início das cirurgias em crianças, era esperada a publicação de um número maior de artigos abordando os resultados a longo prazo do dispositivo de IC na população pediátrica. Segundo Beadie et al. (2005), possíveis justificativas para o número ainda escasso de estudos deste tipo deve-se à problemática metodológica e a forma de apresentação dos resultados mensurados.

Omissão dos dados demográficos ou dos resultados das avaliações para cada usuário, não padronização dos protocolos e medotologia empregada na aplicação dos 


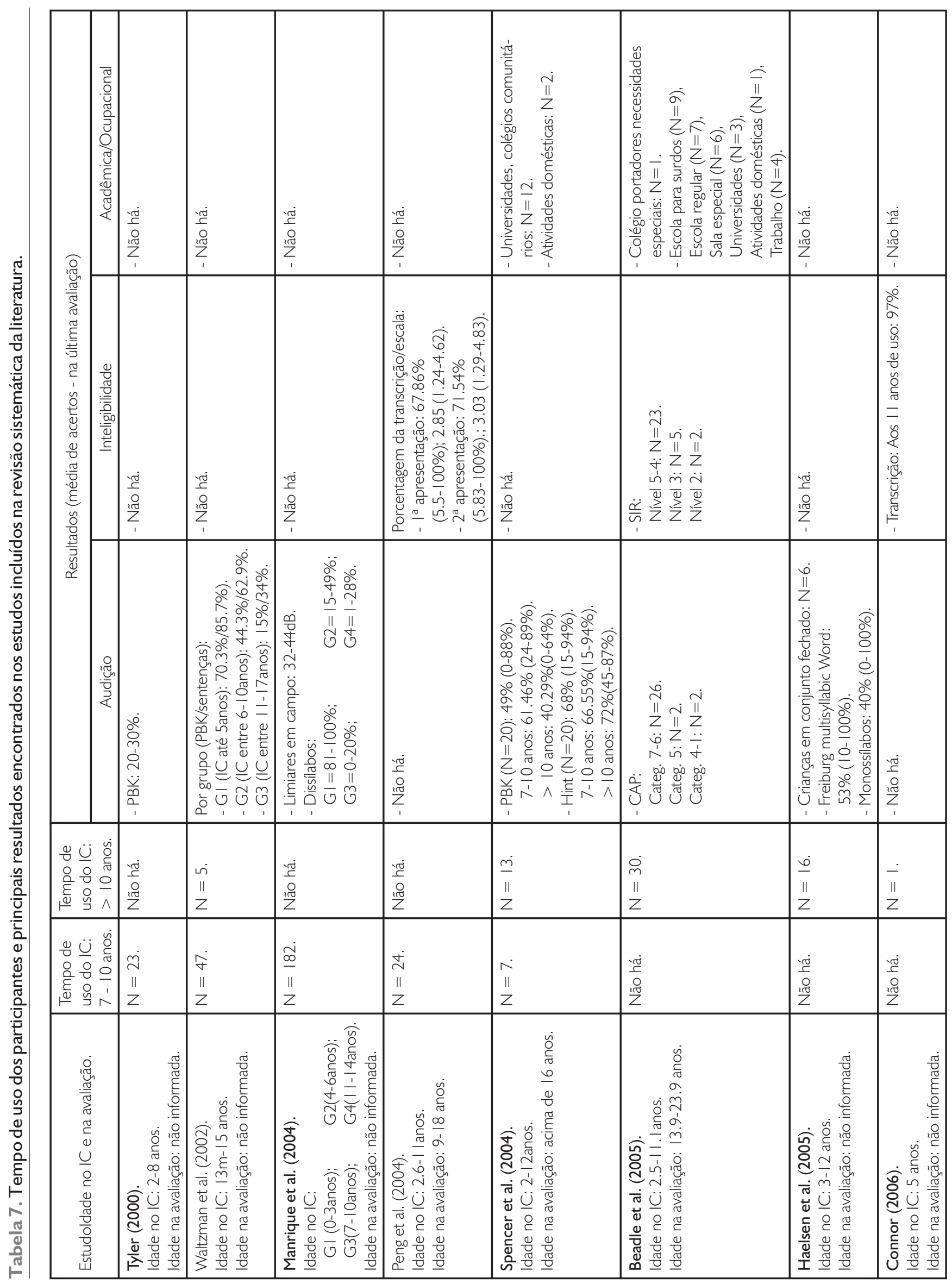




\begin{tabular}{|c|c|c|c|c|c|c|}
\hline 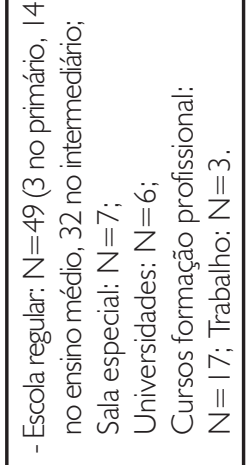 & $\mid$ & 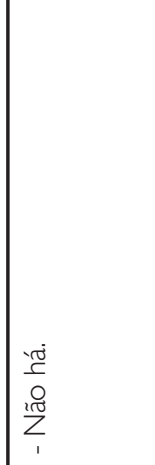 & 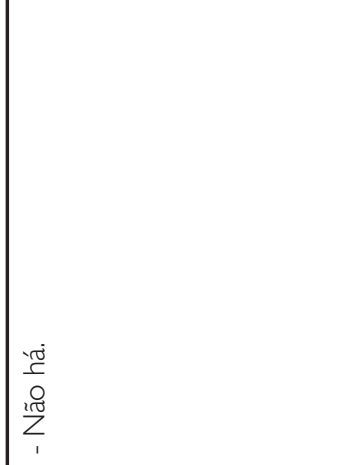 & 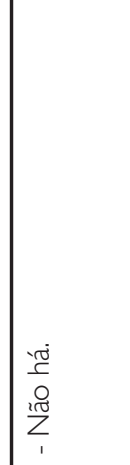 & \begin{tabular}{|l}
$\mid \frac{10}{2}$ \\
$\frac{20}{20}$ \\
$\frac{10}{2}$
\end{tabular} & 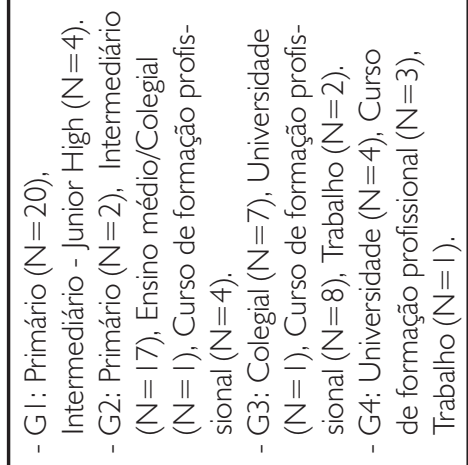 \\
\hline 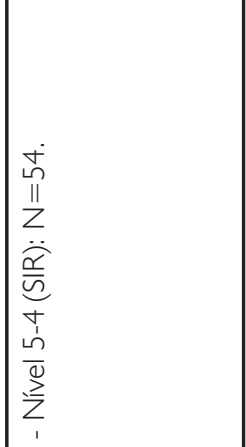 & 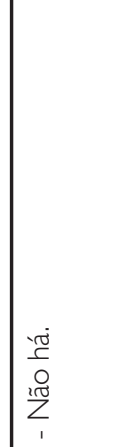 & 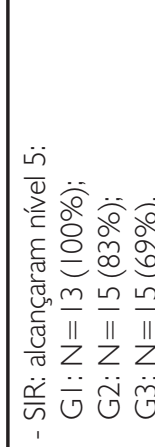 & 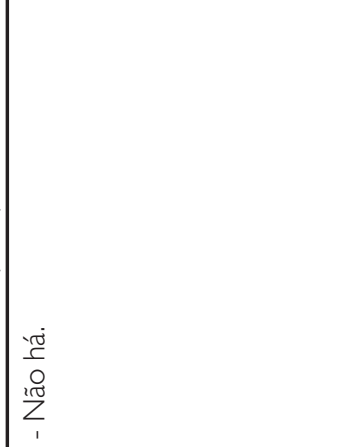 & 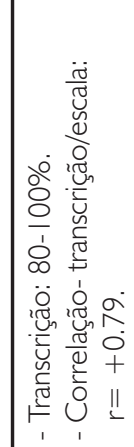 & 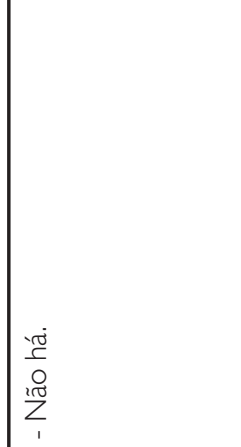 & 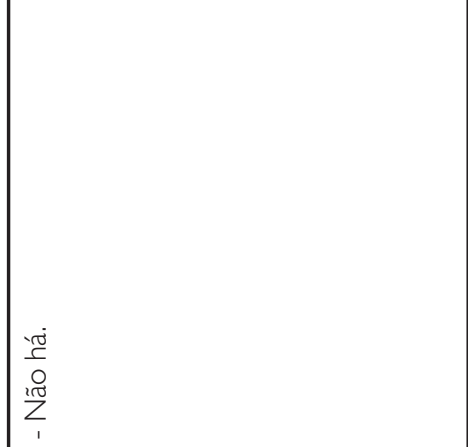 \\
\hline 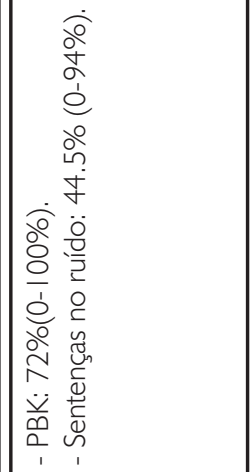 & 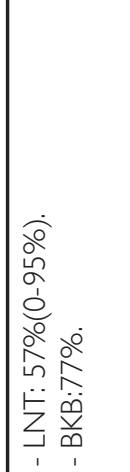 & 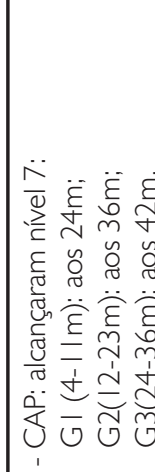 & 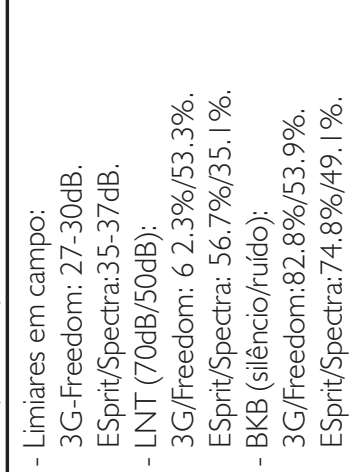 & \begin{tabular}{|l}
2 \\
$\frac{\pi}{2}$ \\
$\frac{\pi}{2}$ \\
$\frac{\pi}{2}$ \\
1
\end{tabular} & 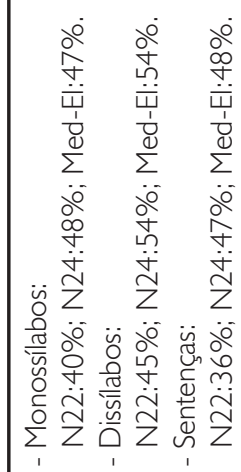 & 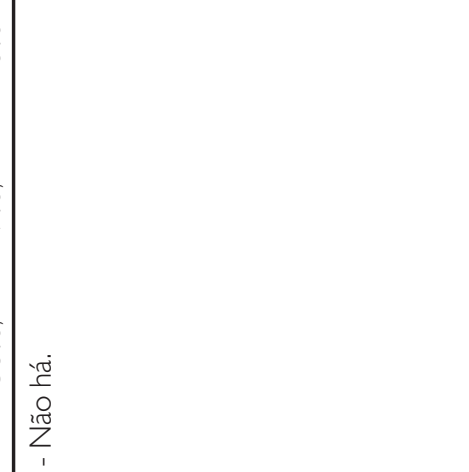 \\
\hline $\begin{array}{l}\text { i } \\
\infty \\
\text { II } \\
z \\
\end{array}$ & $\begin{array}{l}\infty \\
\infty \\
11 \\
z \\
\end{array}$ & 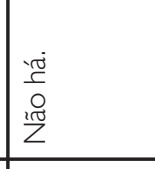 & \begin{tabular}{|l} 
\\
0 \\
11 \\
$z$ \\
\end{tabular} & \begin{tabular}{|l} 
\\
$\pi$ \\
$z$ \\
\end{tabular} & 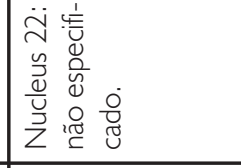 & 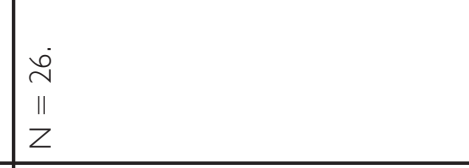 \\
\hline 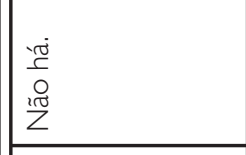 & $\begin{array}{l}\frac{\pi}{2} \\
\text { 要 } \\
\end{array}$ & $\begin{array}{l}\text { Ni } \\
\| 1 \\
z \\
\end{array}$ & $\begin{array}{l}\stackrel{\rho}{n} \\
\| 1 \\
z\end{array}$ & $\begin{array}{l} \\
\text { II } \\
z \\
\end{array}$ & 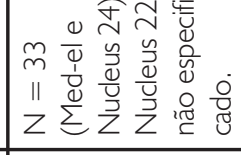 & $\begin{array}{l}\stackrel{ \pm}{ \pm} \\
\text { II } \\
z\end{array}$ \\
\hline 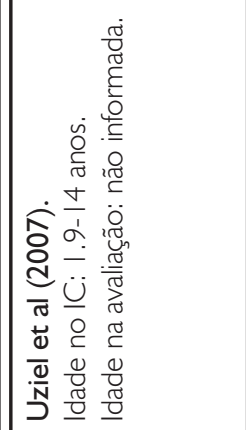 & 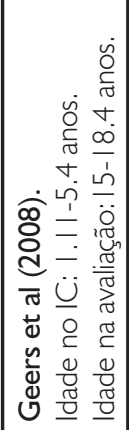 & 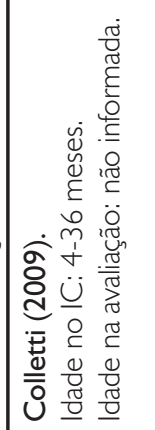 & 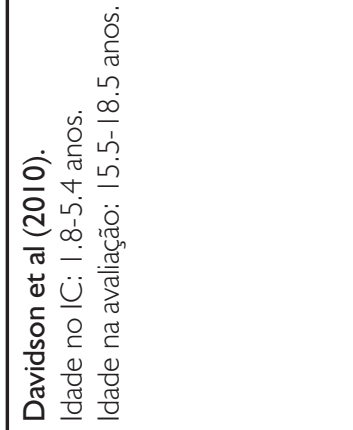 & 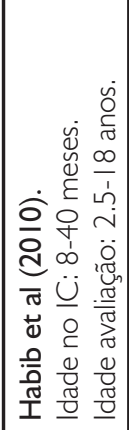 & 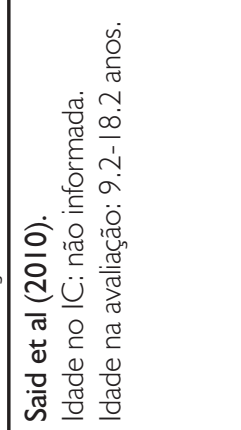 & 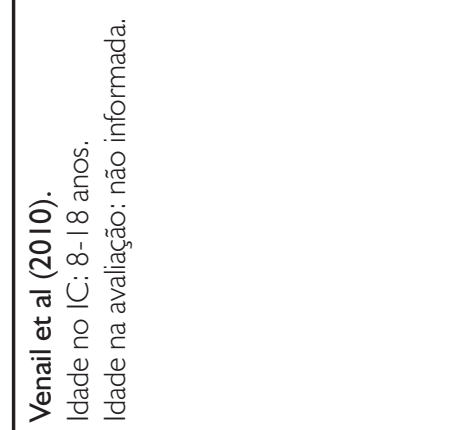 \\
\hline
\end{tabular}


testes, omissão dos usuários com resultados ruins, agrupamento dos usuários em função de diferentes variáveis, problemas com o design do estudo e inadequado follow-up, são alguns dos problemas mais comuns encontrados nos estudos avaliando os usuários de IC ao longo do tempo (Waltzman et al. 2002; Beadle et al., 2005; Venail et al., 2010).

Outro aspecto negativo é que nem todos os estudos abordaram todas as áreas de interesse no acompanhamento dos usuários, tais como, o desenvolvimento das habilidades auditivas, de linguagem oral, a situação acadêmica e ocupacional.

Na presente revisão sistemática, apenas dois estudos (BEAdle et al., 2005 e Uziel et al., 2007) abordaram todos os tópicos dentre as áreas de interesse. A maioria dos estudos abordou apenas uma área de interesse, sendo que seis estudos avaliaram somente as habilidades auditivas (Tyler,2000; Walzman et al., 2002; Manrique et al., 2004; Haelsen et al., 2005; Geers et al., 2008; Davidson et al., 2010; SAID et al., 2010), três estudos analisaram apenas a inteligibilidade de fala (PENG et al., 2004, Connor, 2006; HABIB et al., 2010) e a situação acadêmica e ocupacional foi estudada pelo estudo de VENAIL et al. (2010). Áreas combinadas, habilidades auditivas/inteligibilidade de fala e habilidades auditivas/situação acadêmica foram avaliadas em dois estudos, realizados por COLLETTI (2009) e SPENCER et al. (2004), respectivamente.

Quanto aos procedimentos utilizados, os estudos que avaliaram especificamente as habilidades auditivas aplicaram testes de reconhecimento de palavras monossílabas, sentenças no silêncio/ruído e escala de habilidades auditivas (Tyler,2000; Walzman et al., 2002; MANRique et al., 2004; SPEncer et al., 2004; BeAdle et al., 2005; Haelsen et al., 2005; Uziel et al., 2007; GeErs et al., 2008; Collettr, 2009; Davidson et al., 2010; SAid et al., 2010). Os estudos no idioma espanhol e português utilizaram também listas de palavras dissílabas (MANRIQUE et al., 2004; SAID et al., 2010). Apesar das variáveis metodológicas aplicadas, foi possível observar que a maioria dos participantes destes estudos alcançou níveis complexos de habilidades auditivas. Consequentemente, a maioria dos usuários de IC foi capaz de reconhecer a fala sem o auxílio da leitura orofacial e, portanto, conseguiu concluir os procedimentos de avaliação da percepção da fala em conjunto aberto.

Ainda em relação à avaliação das habilidades auditivas, apenas dois estudos realizados por UzIEL et al. (2007) e DAvidson et al., (2010) mensuraram o reconhecimento de sentenças na presença de ruído (relação S/R +10 dB). Considerando a relevância que a avaliação da percepção da fala na condição de ruído exerce dentro da avaliação dos benefícios e limitações do uso dos dispositivos eletrônicos (Duncan e AarTs, 2006) e, devido ao fato de um número considerável de usuários ter alcançado níveis de habilidades auditivas necessários para o reconhecimento da fala, alcançando um platô nos testes realizados no silêncio, era esperado que um número maior de estudos com a realização de testes de percepção de fala com a apresentação de ruído competitivo.

Seis estudos avaliaram a inteligibilidade de fala após, no mínimo, 7 anos de uso do IC. Novamente, os estudos utilizaram diferentes metodologias. Dentre eles, três utilizaram a escala "Speech Intelligibility Rating" - SIR (BEADLE et al., 2005; Uziel et al., 2007; ColletTi, 2009), um estudo utilizou somente o método da transcrição (CoNNOR, 2006) e dois estudos mensuraram a inteligibilidade da fala pelo método da transcrição juntamente com uma escala de inteligibilidade de cinco níveis (PENG et al., 2004; HaBIB et al., 2010). Houve grande variabilidade nos resultados obtidos por meio do método da transcrição, sendo que as porcentagens, mínima e máxima, obtidas pelo método da transcrição variaram entre $5.5 \%$ a 100\%.

Apesar da variabilidade quantoà inteligibilidade dos usuários de IC, a maioria dos participantes alcançou níveis satisfatórios nas escalas de inteligibilidade. Os resultados sugerem que após 7 anos de uso do IC, a mensagem transmitida oralmente pela maioria das crianças que cresceu usando o dispositivo pode ser compreendida por ouvintes sem experiência com a fala de portadores de deficiência auditiva. No entanto, nem todos os usuários, mesmo após 10 anos de uso do dispositivo, não conseguiramalcançar resultados satisfatórios em transmitir o conteúdo da mensagem por meio da linguagem oral (PENG et al., 2004; Uziel et al., 2007; НАвів et al., 2010).

Em relaçãoaos resultadosacadêmicos e ocupacionais, os estudos coletaram as informações por meio da entrevista com o usuário e/ou familiar. Em geral, as características levantadas pelos autores mostraram que a inserção das crianças em escolas regulares foi aumentando com o tempo de uso do IC e que os usuários alcançaram níveis educacionais satisfatórios. Alguns autores observaram uma tendência dos usuários em acompanhar o padrão acadêmico apresentado pelos seus pais (SPENCER et al., 2004). Apesar da maioria dos participantes frequentar escola regular ou universidade, houve atraso na aquisição das habilidades acadêmicas e foram registrados episódios de repetência escolar, principalmente no ensino intermediário/médio (Spencer et al., 2004; GeErs et al., 2008; Venail et al., 2010).

Houve diferenças importantes entre os estudos relacionadas a o ritmo de desenvolvimento das habilidades comunicativas, aparecimento de platôs no desenvolvimento, bem como variabilidade nos resultados obtidos na última avaliação. Tal variabilidade foi demonstrada em 
todas as áreas avaliadas pelos estudos os autores atribuíram os resultados desta variabilidade, em parte, a existência de determinados fatores.

Presença de audição residual nas frequências graves e compreensão de fala antes da cirurgia de IC foram os fatores associados aos melhores resultados obtidos após anos de uso do IC para Tyler (2000) e Waltzman et al. (2002). O tempo de privação sensorial e a idade na implantação foram as variáveis de maior consenso entre os estudos no que diz respeito aos preditores de sucesso para os usuários de IC. Menor idade na implantação, considerando, especificamente, a faixa etária até dois anos de idade, juntamente com o uso de comunicação oral foram relacionadas ao melhor ritmo de aquisição das habilidades, aos melhores resultados de percepção e de inteligibilidade da fala após longos anos de uso e a tendência de maior proximidade aos resultados obtidos em indivíduos ouvintes. Ao contrário, os piores resultados em todas as áreas avaliadas foram obtidos em usuários com maior privação sensorial (Tyler 2000; Waltzman et al., 2002; MANRique et al., 2004; Peng et al., 2004; Spencer et al., 2004; Geers et al., 2008; Colletti, 2009; Habib et al., 2010; SAID et al., 2010; Venail et al., 2010).

A partir destes resultados, os autores salientaram a importância da realização do IC precocemente, de modo a evitar os períodos de privação sensorial. Os resultados a longo prazo com usuários de IC implantados em diferentes faixas etárias sugerem que, mesmo após anos de uso do dispositivo, as crianças submetidas tardiamente ao IC podem não conseguir alcançar os mesmos resultados das crianças implantadas em idades menores (TyLER, 2000; WaltzMAn et al., 2002; MANRique et al., 2004; SpEncer et al., 2004; Geers et al., 2008; Colletti, 2009; Venail et al., 2010).

Todos os diferentes modelos de dispositivos avaliados proporcionaram benefícios a longo prazo para seus usuários e a diferença entre eles não foi estatisticamente significativa (SAID et al., 2010). No entanto, o uso de diferentes e mais modernas estratégias de codificação de fala foi um aspecto importante discutido em alguns estudos e que contribuiu de modo significativo para a obtenção de melhores resultados tanto nos testes de avaliação das habilidades auditivas, como da inteligibilidade da fala (PENG et al., 2004; Davidson et al., 2010 e SAID et al., 2010).

Os resultados dos estudos também foram positivos quanto ao quesito integridade e funcionamento do dispositivo a longo prazo, mesmo ao longo de dez anos de uso. Segundo os autores, mesmo havendo a necessidade de reoperação, os usuários não apresentaram piora na percepção auditiva após a reimplantação. Dos três estudos que descreveram a ocorrência de falhas no dispositivo interno, houve a necessidade de reimplantação em 9 casos no estudo de Waltzman et al. (2002), 8 casos no estudo de Beadle et al. (2005) e 11 casos no artigo de Uziel et al. (2007).

\section{CONSIDERAÇÕES FINAIS}

Após o levantamento bibliográfico e análise da evidência científica encontrada a partir dos estudos incluídos nesta revisão sistemática da literatura foi possível concluir que existiu um consenso entre os autores dos estudos incluídos na presente revisão sistemática quanto às conclusões a respeito dos benefícios em função do tempo de uso do IC.

Os resultados apresentados sugeriram que o uso a longo prazo do IC proporcionou, a primeira geração de crianças usuárias de IC, significativo progresso e níveis de competência linguística e acadêmica similares aos indivíduos com audição normal da mesma idade. Além, da contribuição do IC para os diversos aspectos inerentes ao processo de desenvolvimento das habilidades comunicativas e acadêmicas e a melhora na qualidade de vida, os resultados dos estudos revelaram a efetividade funcional do IC, consolidando-o como um dispositivo de considerável confiabilidade e durabilidade, mesmo ao longo de dez anos de uso.

Entretanto, houve certa variabilidade nos resultados obtidos após um período superior a 7 anos de uso do IC. Para alguns autores, o maior tempo de privação sensorial e o uso de dispositivos menos avançados tecnologicamente encontrados na primeira geração de crianças implantadas podem ter contribuído de maneira determinante para a existência desta variabilidade.

Estudos a longo prazo com as gerações subsequentes de crianças usuárias de IC são necessários. Espera-se que a nova geração de usuários de Implante coclear, a qual foi submetida ao procedimento cirúrgico em idades menores e com dispositivos com recursos tecnológicos mais avançados, possa alcançar resultados ainda melhores com o tempo de uso do dispositivo.

\section{REFERÊNCIAS BIBLIOGRÁFICAS}

1. Chin SB, Tsai PL, Gao S. Connected speech intelligibility of children with cochlear implants and children with normal hearing. Am J Speech Lang Pathol. 2003, 12(4):440-51.

2. Archbold S, O'Donoghue GM. Ensuring the long-term use of cochlear implants in children: the importance of engaging local resources and expertise. Ear Hear. 2007, 28(2):3S-6S 
3. Richter B, Eissele S, Laszig R, Löhle E. Receptive and expressive language skills of 106 children with a minimum of 2 years experience in hearing with a cochlear implant. Int J Pediatr Otorhinolaryngol. 2002, 64(2):111-25.

4. Calmels MN, Saliba I, Wanna G, et al. Speech perception and speech intelligibility in children after cochlear implantation. Int J Pediatr otorhinolaryngol. 2004, 68:34751.

5. Artières F, Vieu A, Mondain M, Uziel A, Venail F. Impact of early cochlear implantation on the linguistic development of the deaf child. Otol Neurotol. 2009, 30:736-42.

6. Archbold AM, Nikolopoulos TP, Lloyd-Richmond H. Long term use of cochlear implant system in paediatric recipients and factors contributing to non-use. Cochlear Imp Inter. 2009, 10(1):25-40.

7. Nicolas JG e Geers AE. Will they catch up? The role of age at cochlear implantation in the spoken language development of children with severe to profound hearing loss. J Speech Lang Hear Res. 2007, 50:1048-62.

8. Gordon KA, Daya H, Harrison RV, Papsin BC. Factors contributing to limited open-set speech perception in children who use a cochlear implant. Int J Pediatr Otorhinolaryngol. 2000, 56(2):101-11.

9. Robbins AM, Koch DB, Osberger MA, Zimmerman-Phillips S, Kishon-Rabin L. Effect of age at cochlear implantation on auditory skill development in infants and toddlers. Arch Otolaryngol Head Neck Surg. 2004, 130:570-78.

10. Eisenberg LS, Johnson KC, MartinezAS, Cokely CG, Tobey EA, Quittner AL, Fink NE, Wang NY, Niparko JK. CDaCI Investgative Team. Speech recognition at 1-year followup in the childhood development after cochlear implantationstudy: Methods and preliminary findings. Audiol Neurootol. 2006, 11(4):259-68.
11. Wie OB, Falkenberg ES, Tvete O, Tomblin B. Children with a cochlear implant: characteristics and determinants of speech recognition, speech-recognition growth rate, and speech production. Int J Audiol. 2007, 46(5):232-43.

12. Tomblin JB, Peng S, Spencer LJ, Lu N. Long-term trajectories of the development of speech sound production in pediatric cochlear implant recipients. J Speech Language Hearing Research. 2008, 51: 1353-68.

13. Battmer RD, Linz B, Lenarz T. A review of device failure in more than 23 years of clinical experience of a cochlear implant program with more than 3.400 implantees. Otol Neurotol. 2009, 30(4):455-63.

14. Wang NM, Huang TS, Wu CM, Kirk KI. Pediatric cochlear implantation in Taiwan: Long-term communication outcomes. Int JPediatr Otorhinolaryngol. 2007, 71(11):177582.

15. Huber M, Wolfgang $\mathrm{H}$, Klaus A. Education and training of young people who grew up with cochlear implants. Int J Pediatr Otorhinolaryngol. 2008, 72(9):1393-403.

16. Kaplan DMe Puterman M. Pediatric Cochlear Implants in prelingual deafness: medium and long-term outcomes.IMAJ. 2010, 12:107-9.

17. Sampaio RF e Mancini MC. Estudos de Revisão Sistemática: Um guia para síntese criteriosa da evidência científica. Rev Bras Fisiot. 2007, 11(1):83-9.

18. Mendes TM, Alvarenga KF. Capacitação de profissionais da saúde na área de saúde auditiva: revisão sistemática. Rev Soc Bras Fonoaudiol. 2009, 14(2):280-6.

19. Cox RM. Waiting for evidence-based practice for your hearing aid fittings? It's here! The Hearing Journal. 2004, 57(8):10-7. 